\title{
TITLE:
}

\section{Phase transitions in multiplicative competitive processes}

AUTHOR(S):

Shimazaki, H; Niebur, E

CITATION:

Shimazaki, $H$...[et al]. Phase transitions in multiplicative competitive processes. Physical Review E 2005, 72(1): 011912.

ISSUE DATE:

2005-07

URL:

http://hdl.handle.net/2433/50002

RIGHT:

Copyright 2005 American Physical Society 
PHYSICAL REVIEW E 72, 011912 (2005)

\title{
Phase transitions in multiplicative competitive processes
}

\author{
Hideaki Shimazaki \\ Department of Physics, Graduate School of Science, Kyoto University, Kyoto 606-8502, Japan \\ Ernst Niebur \\ Department of Neuroscience, Zanvyl Krieger Mind/Brain Institute, School of Medicine, Johns Hopkins University, \\ Baltimore, Maryland 21218, USA
}

(Received 4 April 2003; revised manuscript received 23 March 2005; published 20 July 2005)

\begin{abstract}
We introduce a discrete multiplicative process as a generic model of competition. Players with different abilities successively join the game and compete for finite resources. Emergence of dominant players and evolutionary development occur as a phase transition. The competitive dynamics underlying this transition is understood from a formal analogy to statistical mechanics. The theory is applicable to bacterial competition, predicting novel population dynamics near criticality.
\end{abstract}

DOI: 10.1103/PhysRevE.72.011912

PACS number(s): 87.23.Kg, 05.45.-a, 03.75.Nt

Competition occurs when two or more players such as organisms, individuals, or companies strive for common but limited resources. It plays a significant role in biological and social activities, and is the basis of evolution. Most natural competition processes allow the introduction of new players, which is a hallmark of an open, nonequilibrium system. In this contribution, we introduce an irreversible discrete multiplicative process with normalization at each time step as a generic model of competition. Players with different abilities successively join the game and compete for finite resources. The model shows macroscopically observable changes in its behavior; at a singularity in the statistical distribution of the players' abilities, certain players become dominant over all others. The emergence of dominant players and the evolutionary development of the system occur as a transition from stationary to nonstationary state of the multiplicative process. We analyze the phase transition in the mathematical framework of Bose-Einstein condensation (BEC), although, of course, systems modeled are classical and not quantum mechanical. The same approach has been applied successfully to models of complex networks [1] and ecosystems [2] that behave analogously to a Bose gas. We show that this approach is applicable to bacterial competition, providing surprising insights and predictions to their dynamics.

Before we present the model, we first introduce a general framework for how our multiplicative competition model is related to a statistical mechanics concept. Let $\varphi(\epsilon)$ be a function that satisfies the following conditions for an arbitrary $C^{2}$ density function $g(\epsilon)(\geqslant 0)$ defined on $\epsilon \in\left[0, \epsilon_{\max }\right]$ :

$$
\begin{gathered}
\int d \epsilon g(\epsilon)=1, \\
\int d \epsilon g(\epsilon) \varphi(\epsilon)=m+y_{0}, \\
\int d \epsilon g(\epsilon) e^{-\beta \epsilon} \varphi(\epsilon)=M .
\end{gathered}
$$

All terms on the right-hand sides of these equations and $\beta$ in Eq. (3) are positive constants. Then $\varphi(\epsilon)$ is given by

$$
\varphi(\epsilon)=\frac{y_{0}}{1-e^{-\beta \epsilon-\alpha}},
$$

where $e^{-\alpha}=m / M$. For, dividing Eq. (2) by $y_{0}$, then subtracting Eq. (1), yields

$$
\int \operatorname{d\epsilon g}(\epsilon) \frac{\varphi(\epsilon)-y_{0}}{y_{0}}=N,
$$

where $N=m / y_{0}$. Multiplying Eq. (3) by $N / M$ and subtracting Eq. (5) from the result, the fundamental lemma of the calculus of variation then yields Eq. (4). Hence, the so-called occupation number in Eq. (5),

$$
n(\epsilon)=\frac{\varphi(\epsilon)-y_{0}}{y_{0}}=\frac{1}{e^{\beta \epsilon+\alpha}-1},
$$

becomes the Bose distribution. From Eq. (4), such a function $\varphi(\epsilon)$ may be obtained from the sum of a geometric progression with ratio $e^{-\beta \epsilon-\alpha}<1$. This motivates the analysis of the following multiplicative process.

The competition we introduce is defined by three conditions at each time step. (i) Players compete for a fixed total amount of resources. (ii) The resource gained by a player is proportional to the player's innate ability and to its resource gained at the previous time step. (iii) New players join the game, each with the same initial resources. The only exception is the first player (pioneer), who starts the game with all the resources available. These rules are summarized in a simple multiplicative process

$$
y_{i}(t+1)=a_{i} b(t) y_{i}(t),
$$

where $y_{i}(t)$ is the gain of the $i$ th player at time $t$ and $a_{i}$ is its ability, a positive and time-independent random variable chosen from a distribution $\rho(a)$. The term $b(t)$ is a normalization factor, which models the limited resources. For simplicity, we first assume that $l$, the number of newly introduced players at every time step, is 1 . This means we consider $t+1$ simultaneous equations $(i=0, \ldots, t)$ at time $t$. The normalization factor is then defined by $b(t)=m / M_{t}$, where $M_{t}$ is given by 


$$
M_{t}=\sum_{j=0}^{t} a_{j} y_{j}(t) .
$$

This assures that the amount of resources distributed among $t$ players at time $t$ is $m$ for $t>0$. The initial value of a new player is $y_{i}(i)=y_{0}$, except for the pioneer whose initial value is $y_{0}(0)=m+y_{0}$. Therefore, the total resources distributed among all players are limited to $m+y_{0}$ at every time step. Due to the normalization, we let $a \in\left[a_{\min }, 1\right]$, where $a_{\text {min }}$ $>0$, without loss of generality.

We now consider the time evolution of players except for the pioneer. The gain of the $i$ th player at time $t$ is given by $y_{i}(t)=\Omega_{i}(t) y_{0}$, where

$$
\Omega_{i}(t)=a_{i}^{t-i}\left(\prod_{t^{\prime}=i}^{t-1} b\left(t^{\prime}\right)\right) .
$$

The dynamics given by $\Omega_{i}(t)$ shows distinct phases determined by the shape of the ability distribution. To see this, we parametrize $\rho(a)$ by the inverse temperature $\beta(=1 / T)$ through $a=e^{-\beta \epsilon}$. The random variable $\epsilon \in\left[0, \epsilon_{\max }\right]$ is chosen from $g(\epsilon)$, a state density function which now defines the system. We now systematically change $\rho(a)[=g(\epsilon)|d \epsilon / d a|]$ by fixing $g(\epsilon)$ and changing $\beta$. If $g(\epsilon)$ is a monotonically increasing function, which we will assume, high temperatures mean that the distribution of the players' abilities is restricted to a small range-i.e., all players have relatively similar abilities-while low temperature is synonymous with occasional appearance of superior players relative to others. From Eq. (9), we have

$$
\ln \Omega_{i}=(t-i)\left\{-\beta \epsilon_{i}-\langle\ln b\rangle\right\},
$$

using $\ln \Pi_{t^{\prime}=i}^{t-1} b\left(t^{\prime}\right)=\sum_{t^{\prime}=i}^{t-1} \ln b\left(t^{\prime}\right) \sim(t-i)\langle\ln b\rangle$. By assuming stationarity of $M_{t}$, discussed below, we let its time average be

$$
\langle\ln b\rangle=-\alpha,
$$

where $\alpha$ is a time-independent constant. From Eqs. (10) and (11), we obtain $y_{i}(t)=e^{\left(-\beta \epsilon_{i}-\alpha\right)(t-i)} y_{0}$. Given $e^{-\beta \epsilon_{i}-\alpha}<1$, the cumulative gain $\varphi_{t}\left(\epsilon_{i}\right)=\sum_{t^{\prime}=i}^{t} y_{i}\left(t^{\prime}\right)$ converges to $\varphi\left(\epsilon_{i}\right)$ in Eq. (4). Hence, the normalization of $\varphi_{t}\left(\epsilon_{i}\right)$ by the initial value $y_{0}$, $n_{t}\left(\epsilon_{i}\right)=\left\{\varphi_{t}\left(\epsilon_{i}\right)-y_{0}\right\} / y_{0}$, approaches the Bose distribution in the thermodynamic limit $(t \rightarrow \infty)$. Indeed, in this limit,

$$
\lim _{t \rightarrow \infty} M_{t}=\int d \epsilon g(\epsilon) \sum_{t^{\prime}=0}^{\infty} e^{-\beta \epsilon} y\left(t^{\prime}\right),
$$

where $\sum_{t^{\prime}=0}^{\infty} y\left(t^{\prime}\right)=\varphi(\epsilon)$. As the assumption of stationarity yields $\lim _{t \rightarrow \infty} \ln b(t)=\langle\ln b\rangle$, we have $\lim _{t \rightarrow \infty} m / M_{t}=e^{-\alpha}$ from Eq. (11). By substituting this into Eq. (12), we obtain a self-consistent equation

$$
\int \operatorname{d\epsilon g}(\epsilon) \frac{1}{e^{\beta \epsilon+\alpha}-1}=N,
$$

where $N=m / y_{0}$. Note that we can generalize Eq. (7) such that we allow $l(\geqslant 1)$ new players to join the competition with initial value $y_{0}$ at each step. From a similar argument, we obtain Eq. (13), where $N=m / y_{0} l$. Thus the deduced form,
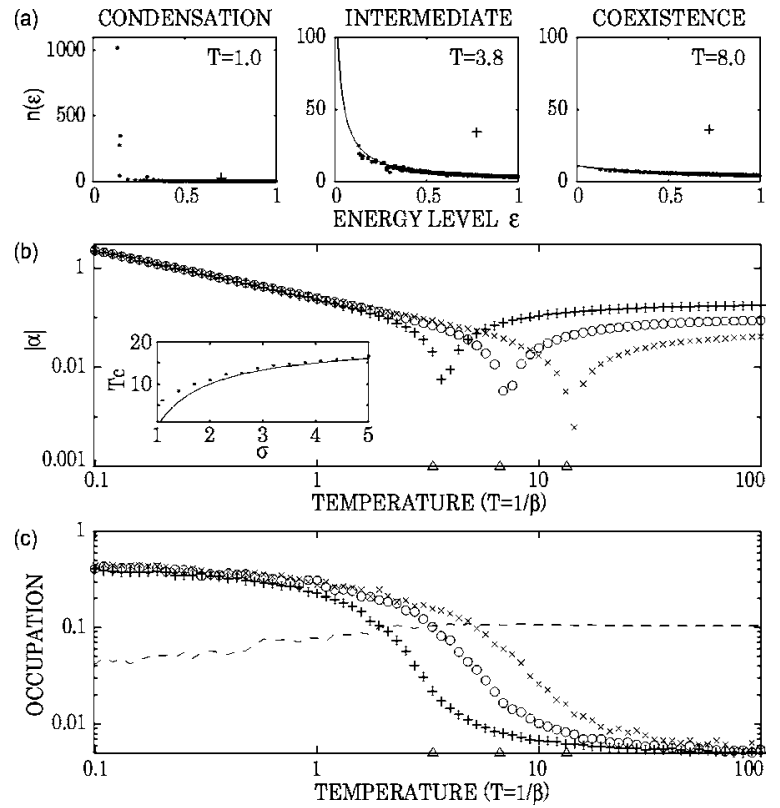

FIG. 1. (a) Normalized cumulative gain $n_{t}\left(\epsilon_{i}\right)$ for $m=5$ and $T$ $<T_{c}$ (left), $T \approx T_{c}$ (center), and $T>T_{c}$ (right). The solid line is the Bose distribution with $\alpha$ from Eq. (11). The plus sign indicates a pioneer. Plots of the last ten entrants were excluded. (b) Numerical calculation of $|\alpha|$ with $\alpha$ from Eq. (11), averaged over 500 trials. Symbols,$+ \bigcirc$, and $\times$ indicate $m=5,10$, and 20. Change in the sign of $\alpha$ indicates the transition. Triangles on the abscissa are the transition temperatures from Eq. (17); $T_{c} \approx 3.33,6.67$, and 13.3. Shown in inset is the dependence of $T_{c}$ on exponent $\sigma$ for $m=20$ (dots) and the analytical solution from Eq. (17), solid line. (c) Cumulative occupation by the most capable player was calculated from $\varphi_{t}\left(\epsilon_{\min }\right) / \Sigma_{j} \varphi_{t}\left(\epsilon_{j}\right)$ at $t=200$. Symbols as in (b). Dashed line, occupation by pioneer for $m=20$. In all simulations, $y_{0}=1, l=1, \sigma=3$, and $\epsilon_{\max }=1$.

Eq. (13), of the competition under stationarity assumption shows a formal analogy to those obtained for a quantum gas. The normalization factor $\alpha$ plays the role of keeping the total resources gained by all players constant at every time step, in analogy to the chemical potential of a quantum gas which is introduced for the conservation of particle number. According to this reasoning, condensation of resources to a single player analogous to BEC is expected at low temperature where $\alpha$ vanishes.

To study this prediction, we simulate the multiplicative process, Eq. (7), adopting a standard density function

$$
g(\epsilon)=C_{\sigma} \epsilon^{\sigma-1}
$$

where $C_{\sigma}=\sigma / \epsilon_{\max }^{\sigma}(\sigma>1)$. We find two distinct phases for the distribution of the normalized cumulative gain $n_{t}\left(\epsilon_{i}\right)$ $=\left\{\varphi_{t}\left(\epsilon_{i}\right)-y_{0}\right\} / y_{0}$. At high $T, n_{t}\left(\epsilon_{i}\right)$ obeys the Bose distribution [Fig. 1(a)]. At low $T$, the coordinated distribution breaks down: players with low energy (high ability) dominate a large fraction of the cumulative gain. This condensate exists only below a critical point $\alpha=0$. For, if $\alpha$ is negative, $\varphi\left(\epsilon_{i}\right)$ of the player with $\epsilon_{i}<-\alpha / \beta$ does not converge. Its normalized cumulative gain cannot be incorporated into the integral in Eq. (13); rather, it has to be added as an extra term. The 
critical temperature for this transition can be predicted in the usual way: Change of variables $y=\beta \epsilon$ and use of $\alpha=0$ in Eq. (13) yields

$$
N=\frac{\sigma}{\left(\beta \epsilon_{\max }\right)^{\sigma}} \int_{0}^{\beta \epsilon_{\max }} \frac{y^{\sigma-1}}{e^{y}-1} d y .
$$

By approximating the upper limit of integral in Eq. (15) by infinity, the critical temperature $T_{c}\left(=1 / \beta_{c}\right)$ is given by

$$
T_{c}^{(1)} \sim \epsilon_{\max }\left\{N^{-1} \Gamma(\sigma+1) \zeta(\sigma)\right\}^{-1 / \sigma},
$$

for $N \ll 1$. When $N \gg 1$, this approximation is not valid because of the high critical temperature; instead, from $e^{y} \simeq 1$ $+y$ and Eq. (15), one obtains

$$
T_{c}^{(2)} \sim \epsilon_{\max }\left(1-\sigma^{-1}\right) N .
$$

The transition can be seen in the occupation by the most capable player, defined by $\varphi_{t}\left(\epsilon_{\min }\right) / \Sigma_{j} \varphi_{t}\left(\epsilon_{j}\right)$. As $T$ decreases below $T_{c}$, its occupation dramatically increases, supporting the prediction [Fig. 1(c)].

We verified the existence of a BEC analog in a discrete multiplicative process, as was shown in a continuous model [1]. However, we emphasize that, as a matter of principle, our classical dynamical system is not equivalent to a quantum gas. The most important difference is based on the following observation. The time evolution of each player's gain is different above and below the predicted $T_{c}$. Above $T_{c}$, the gains of all players monotonically decrease. Below $T_{c}$, not all of them show monotonic behavior, and the competitive dynamics is disordered. The observed nonequilibrium phase transition from the ordered to disordered state occurs as a violation of the stationarity in weighted mean ability, $M_{t} /\left(m+y_{0}\right)$. It is stationary if the gain of all players monotonically decreases to zero, which allows the argument below Eq. (10). Otherwise, if the gain of one player rises to dominate the resources, the weighted mean ability approaches the ability of this one dominant player. Then, due to the replacement of the dominant player upon the entrance of a player with higher ability, we observe an irreversible increase of the weighted mean ability, indicating that the system is now evolving. Thus dominance and evolution are aspects of nonstationary dynamics. We emphasize that the phase transition yielding evolution does not happen in equilibrium systems.

We now consider application of the theory to the competition of clonal strains of asexual Escherichia coli serially propagated on glucose-limited medium. The population dynamics is most suitably described by a stochastic branching process with mutation and selection. Consider the $i$ th strain with fitness $a_{i}$, mutation rate $\eta$, and population size $y_{i}(t)$. Let $y_{i}(t+1)[i=1, \ldots, Q(t)]$ be given by a Poisson distribution with mean $\lambda_{i}=\widetilde{m}(1-\eta) a_{i} y_{i}(t) / \Sigma_{i=0}^{Q(t)} a_{i} y_{i}(t)$. Here $Q(t)$ is the number of mutants that were generated and that survived the initial step since the process started. The number of mutants produced at time $t, Q(t+1)-Q(t)$, is drawn from a Poisson distribution with mean $l=\tilde{m} \sum_{j=0}^{Q(t)} \eta a_{j} y_{j}(t) / \Sigma_{i=0}^{Q(t)} a_{i} y_{i}(t)$. Note that the average total number of cells at time $t$ is fixed to $\sum_{i=0}^{Q(t)} \lambda_{i}+l=\tilde{m}$ due to the normalization factor in the above equations.
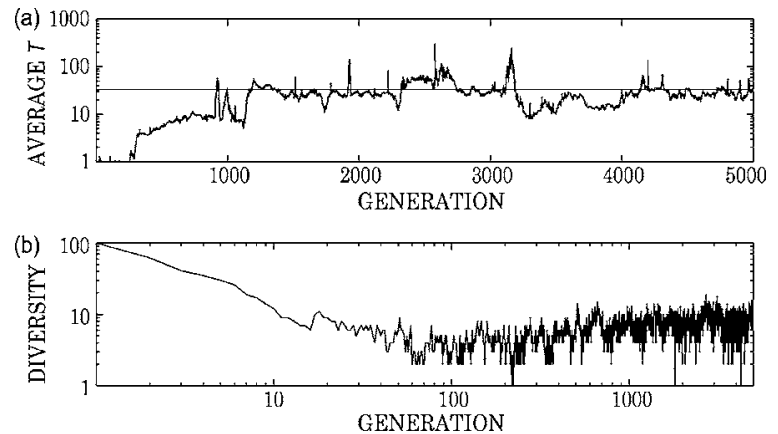

FIG. 2. A stochastic branching process with mutation and selection described in the text approaches a critical state by natural selection. Average total population is $\tilde{m}=100$. Simulation started with 100 unique strains whose fitness is drawn from a fitness distribution with $T=1, \sigma=3 / 2$, and $\epsilon_{\max }=1$. The $i$ th strain produces mutants (mutation rate $\eta=0.01$ ) whose fitness is drawn from a fitness distribution with $T_{i}$ given by Eq. (19) $(\gamma=2)$. (a) Average temperature of the system stays near the transition point $T_{c}=33$. In early stages, the average temperature increases, indicating initial adaptive evolution. (b) Number of existing strains at each steps (diversity) decreases during initial adaptive evolution. However, further dominance by a few species is suppressed as the system approaches near criticality.

It is clear that our process, Eq. (7), is a deterministic approximation of this stochastic population dynamics. A monotonically decreasing fitness distribution $\rho(a)$ should be used because most mutations are likely to be deleterious [3]. We thus decided to use the same $\rho(a)$ used in the analysis of the deterministic model [and the state density Eq. (14)] because it satisfies this basic tenet. Our results do not depend, however, on the precise form of the state density; other parametrizations of the fitness distribution of $\rho(a)$ such as the Beta distribution defined on $a \in[0,1]$ yield essentially the same results (not shown).

Routes to adaptive evolution. A strong prediction of the theory is the existence of a singular point on the emergence of evolution. We observed the transition from stationary to nonstationary state in the numerical simulation of the stochastic process by decreasing the temperature $T$. The transition point is predictable from the critical temperature $T_{c} \mathrm{ob}-$ tained by the deterministic theory [Eq. (17)]. Above $T_{c}$, dominance by a capable player (strain) appears. Below $T_{c}$, the dynamics are governed by the random drift of dominant strains. The random fluctuation of dominant strains is the most striking difference from dynamics of the deterministic model.

Another route to generate an evolutionary development is to increase $T_{c}$ by fixing $T$. The critical temperature given by Eq. (17) is proportional to $N$, which is related to mutation rate through $\eta=l /(m+l)=(N+1)^{-1}$. We thus obtain

$$
T_{c} \sim \epsilon_{\max }\left(1-\sigma^{-1}\right)\left(\eta^{-1}-1\right) .
$$

Given $T$, decreasing $\eta$ increases $T_{c}$ such that $T<T_{c}$ is achieved. A sufficiently low mutation rate not only prevents deleterious offspring but is necessary for adaptive evolution 
itself. The deleterious role of high mutation rate to adaptive evolution termed "clonal interference" was observed in experiments of E. coli competition [4] and theoretically investigated elsewhere [5].

Approach to the transition point by natural selection. An important question is whether $T$ is comparable to $T_{c}$ under natural conditions. Evolutionary progress observed in laboratory experiments of $E$. coli competition shows alteration of long periods of relative stasis with short bursts of rapid change caused by rare beneficial mutations [6]. Therefore, empirically observed dynamics is consistent with model behavior at $T<T_{c}$ in our construct at least during early stages of competition. However, we argue that there exists a biologically plausible scenario in which $T$ may eventually become comparable to $T_{c}$ by internally tuning the parameter $[7,8]$.

Instead of using a common fitness distribution for all strains, it is physiologically plausible to assume that each strain has its unique fitness distribution. We assume that the fitness of mutants originating from the $i$ th strain of fitness $a_{i}$ is drawn from a fitness distribution characterized by the temperature $T_{i}=1 / \beta_{i}$. Since most mutants are deleterious, the average fitness produced with $\beta_{i}$ should be less than $a_{i}$ [i.e., $\langle a\rangle=e^{-\beta_{i}\langle\varepsilon\rangle}\left\langle a_{i}\right.$, where $\langle\varepsilon\rangle=\varepsilon_{\max } \sigma /(\sigma+1)$ for the state density Eq. (14)]. Henceforth, the inverse temperature $\beta_{i}$ is given by

$$
\beta_{i}=-\gamma\langle\varepsilon\rangle^{-1} \ln a_{i},
$$

where $\gamma>1$ to satisfy $\langle a\rangle<a_{i}$.

At the beginning of adaptive evolution, strains with higher fitness are chosen by natural selection. Dominance by strains with high fitness increases the average temperature of the population. Suppose that adaptive evolution achieves a neutral condition $T>T_{c}$. It is then by chance whether or not a certain strain is picked up. Since a dominant strain is prone to produce mutants inferior to the dominant strain itself, those deleterious strains are likely to be picked up and the average temperature decreases. There are ever-going cycles of adaptive evolution, neutral state, then collapse of the dominance (Fig. 2). The advantage of the dynamics that approaches criticality is rather clear. It allows initial adaptive evolution, permanently eliminating unfavorable genotypes, but then significantly slowing down or preventing further evolution and dominance: close to the critical state, strains can coexist for a substantial period of time. Diversity introduced by the dynamics near criticality is clearly advantageous for the whole ecosystem, which is exposed to global environmental changes. We thus conjecture that this strategy might be taken by some haploid species.

We thank H.G. Schuster and S. Shinomoto for very helpful comments. This work was supported by the Murata Overseas Scholarship Foundation (H.S.) and NIH Grant No. R01 NS43188-01A1 (E.N.).
[1] G. Bianconi and A. L. Barabasi, Phys. Rev. Lett. 86, 5632 (2001).

[2] I. Volkov, J. R. Banavar, and A. Maritan, Phys. Rev. Lett. 92, 218703 (2004).

[3] R. A. Fisher, The Genetical Theory of Natural Selection (Dover, New York, 1958).

[4] J. A. G. M. de Visser, C. W. Zeyl, P. J. Gerrish, J. L. Blanchard, and R. E. Lenski, Science 283, 404 (1999).
[5] P. J. Gerrish and R. E. Lenski, Genetica (The Hague, Neth.) 103, 127 (1998).

[6] S. F. Elena, V. S. Cooper, and R. E. Lenski, Science 272, 1802 (1996).

[7] P. Bak, C. Tang, and K. Wiesenfeld, Phys. Rev. Lett. 59, 381 (1987).

[8] S. A. Kauffman and S. Johnsen, J. Theor. Biol. 149, 467 (1991). 